\title{
Menstrual Cycle in Female Workers
}

\author{
Thu Huong Nguyen \\ Faculty of Public Health, Hue University of Medicine and Pharmacy, Vietnam \\ Received: August 2, 2020 \\ Received in Revised: August 10, 2020 \\ Accepted August 13, 2020
}

\begin{abstract}
Menstrual disorders are abnormalities that occur in the menstrual cycle. There are various menstrual disorders that women can experience, ranging from too little or too much menstrual blood, painful menstruation, to depression before menstruation or premenstrual dysphoric disorder. Physical activity is divided into three levels, namely light, moderate and heavy physical activity. Physical activity of heavy intensity can cause physiological disorders of the menstrual cycle. presence of menstruation (amenorrhoea), thinning of the bones (osteoporosis), menstrual irregularities or intermenstrual bleeding, abnormal growth of the uterine wall, and infertility.
\end{abstract}

Keywords: Female Worker, Menstruation, Cycle.

\section{Introduction}

In the dictionary of health terms, menstruation or menstruation is an event of monthly menstrual discharge, which accompanies the release of eggs from the ovaries from the womb (womb or uterus) of women. Menstruation is said to be normal if a menstrual cycle is obtained, not less than 24 days, but not more than 35 days, the length of menstruation is three to seven days, with the amount of blood during menstruation not exceeding $80 \mathrm{~mL}$, change sanitary napkins two to six times per day. The menstrual cycle in women starts from the first day of bleeding. While the cycle ends right before entering the next menstruation.

Describing the menstrual cycle is a complex process that includes both reproductive and endocrine. The menstrual cycle is a complex series of events that influence each other and occur simultaneously (Iyibozkurt, 2011; Norman, 2008). The menstrual cycle is the distance between the start date of the last menstruation and the start of the next menstruation. The normal menstrual cycle length or considered the classic menstrual cycle is 28 days plus or minus 2-3 days.

Eumenorrhea is a regular menstrual cycle with bleeding intervals that occur between 21-35 days (Bushman et al, 2006; Kissel et al, 2014; Loucks et al, 1992). Polimenorrhea, namely menstruation that often occurs and is abnormal. Oligomenorrhea, menstrual cycles with an interval of 35-90 days, the amount of bleeding may be the same, the cause is hormonal disorders. Amenorrhea, which is a delay in menstruation for more than three consecutive months or no menstruation occurs.

Metroragia can be caused by hormonal conditions and anatomical abnormalities. In hormonal disorders, there are disorders of the hypothalamus-pituitary axis, ovaries, and stimulation of estrogen and progesterone in the form of bleeding that occurs outside menstruation, in the form of spotting and continuous, and prolonged menstrual bleeding. The menstrual cycle is regulated by certain hormones (Zhao et al, 2015; Huang et al, 1996; Arrais \& Dib, 2006). The body 
hormones that function to regulate the menstrual cycle are follicle stimulating hormone and luteinizing hormone. These hormones are produced by the pituitary gland. Besides being able to regulate the menstrual cycle, this hormone also plays a role in stimulating the ovaries to stimulate progesterone and also estrogen. Estrogen and progesterone themselves serve to prepare the uterus and breasts in case of fertilization.

Pathological conditions associated with Menstruation can be in the form of tension before menstruation (premenstrual tension), a complaint that starts about a week before and after menstruation. Occurs due to an imbalance of estrogen and progesterone before menstruation. Women also experience irregular menstrual cycles. This means that the menstrual cycle they have can be longer or shorter than the menstrual cycle in general. Usually irregular cycles with the longest periods occur at the beginning of menstruation (menarche) and also before menopause occurs.

\section{Anatomy of the Female Reproductive Organs}

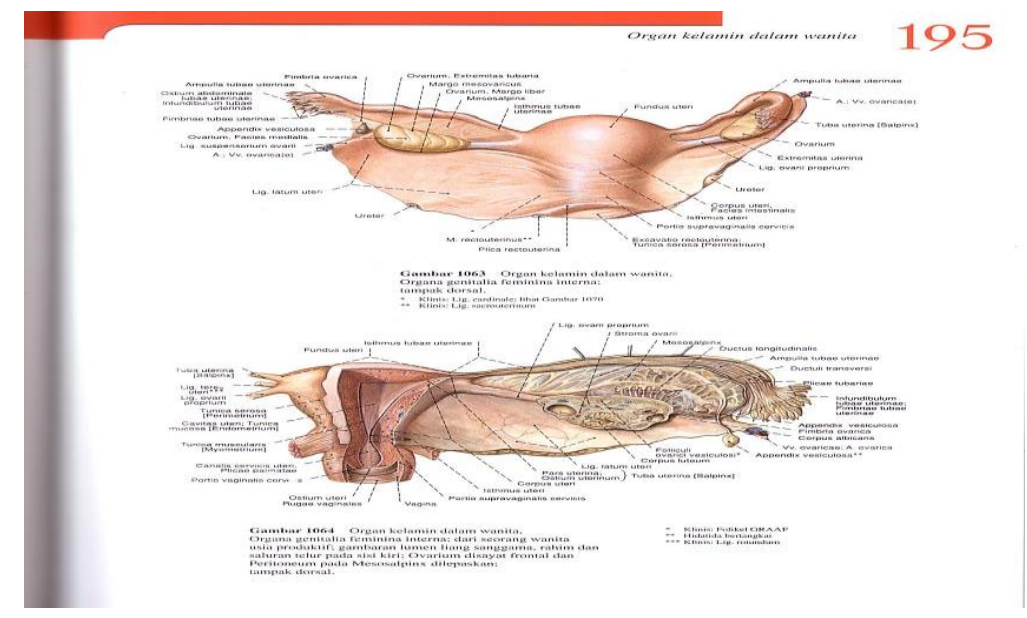

Figure 1. Female reproductive organ

\section{Menstrual Cycle Physiology}

Menstruation is closely related to the factors that affect ovulation, if the ovulation process is regular, the cycle is regular. The follicular phase starts on the first day of menstruation. At the beginning of this phase, the endometrium is thick and rich in fluids and nutrients designed for nutrition for the embryo. If there is no fertilized egg, the levels of estrogen and progesterone are low, so that the upper lining of the uterus, which is the endometrium, sheds and menstrual bleeding occurs.

The normal menstrual cycle length is 21-35 days, usually 28 days. The cycle is permanent and regular at the age of 18-40 years. Mean blood loss is $40-50 \mathrm{ml}$, of which $70 \%$ is lost in the first 48 hours, and contractions are strongest in the first 24-48 hours. 9 At the same time, the pituitary gland slightly increases FSH production. This hormone then stimulates the growth of 3-30 follicles, each follicle contains an egg. The end of the phase. Usually only one follicle develops, called a de Graaf follicle. This follicle then immediately produces estrogen and estrogen which suppresses FSH production. So that the anterior pituitary lobe releases the second gonadotropin hormone, namely LH. 
The mature de Graaf follicles contain lots of estrogen and cause the endometrium to grow and proliferate. In some references this is called the proliferation phase. The follicular to proliferation phase lasts 13-14 days and is the longest phase of this phase to shorten as menopause approaches. This phase ends right at LH increased suddenly. The ovulation phase begins when de Graaf's follicle becomes more mature, approaching the ovary under the influence of LH. After that the follicle develops and an egg (ovum) is released from the ovary (ovulation). In this ovulation sometimes there is a slight bleeding that stimulates the peritoneum in the pelvis, so that There is a pain called intermenstrual pain (Mitteoxhmerz). Here, the endometrium continues to proliferate to form indentations.

The ovulation phase usually lasts 16-32 hours, ending after the release of the ovum. About 12-14 hours later, there is a surge in $\mathrm{LH}$ production that can be measured from the urine. This measurement can simultaneously determine whether a woman is fertile. The eggs can be fertilized for only up to 12 hours after release. Fertilization is more if sperm is in the reproductive tract before the ovum.

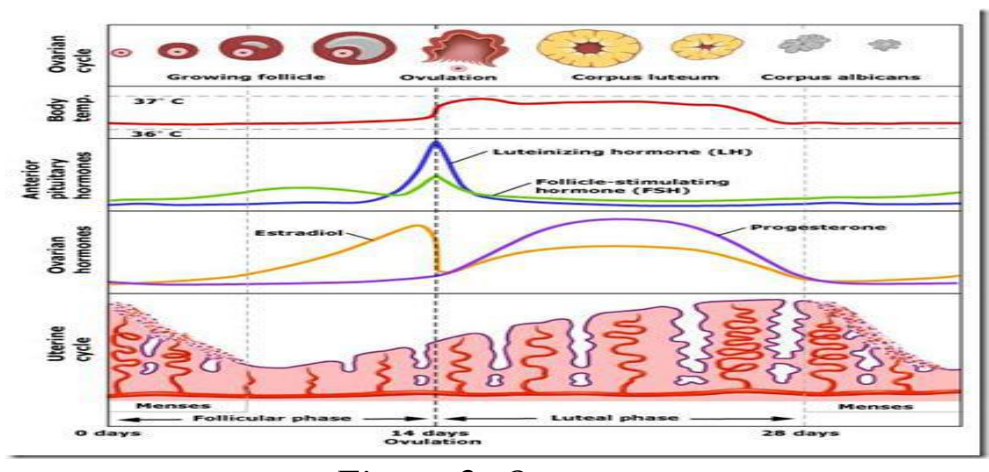

Figure 2. Ovum

\section{Menstrual Cycle Disorders}

After understanding the normal menstrual cycle with menarche as the starting point, several menstrual disorders can be identified. Hypermenorrhea is a form of menstrual cycle disorder that remains regular, the amount of blood that is released is quite large and can be seen from the pads used and blood clots. Hypomenorrhea is a form of abnormalities in the menstrual cycle that remains regular according to the menstrual schedule, the amount is small, with the fact that it does not bleed much.

Approaching menstruation, not a few women experience mild abdominal pain or cramps, headaches, and psychological complaints, such as mood changes, feeling anxious, restless, and irritable. The symptoms that appear closer to menstruation are called PMS or premenstrual syndrome.

\section{Overview of Physical Activity}

Physical activity is divided into three levels, namely light, moderate and heavy physical activity. Mild physical activity is anything related to moving the body, for example walking, table tennis, golf, typing, cleaning rooms, and shopping. Moderate physical activity is movement of the body that causes a significant expenditure of energy that causes breathing to be slightly faster than normal, for example cycling, skiing, dancing, tennis, and climbing stairs. Meanwhile, strenuous physical activity is body movement that causes considerable energy expenditure (calorie 
burning) so that breathing is much faster than usual, for example basketball, soccer, swimming, and weightlifting.

There are four main dimensions that are the focus of measuring physical activity, namely type, frequency, duration and intensity. Type is a type of physical activity that is carried out such as sitting, standing, walking, cycling, etc .; physical activity frequency refers to the number of physical activity sessions per unit time; duration of physical activity is the length of time spent doing physical activity; and the intensity of physical activity is often expressed in terms of light, moderate (moderate), or heavy (vigorous)

Several studies have noted that menstrual disruption occurs during the period of employment (Côté et al, 2002; Ng et al, 1992; Nohara et al, 2010; Su, 2008) where weight loss is significantly more at risk of delayed menstruation than female workers who maintain their weight during the testing period. Increasing such exercise affects menstruation only when accompanied by weight loss. A hypothetical mechanism by which exercise will affect the menstrual cycle by involving the hypothalamus-pituitary-adrenal axis (Carbon in Saadiah).

\section{Review of the Relationship between Physical Activity and Female worker's Menestuation Cycle}

duration of use of contraception has a relationship with the incidence of menstrual disorders in female workers. Female workers who have poor nutrition can cause an imbalance in FSH and LH hormones so that the menstrual cycle can become irregular. Menarche or age at first menstruation is associated with dysmenorrhea, whereas Menarche tarda is generally associated with heredity.

The use of contraceptives, the type of contraception and the duration of use were also associated with the occurrence of pain during menstruation. The use of hormonal contraceptives according to WHO can cause excessive bleeding during menstruation and menstrual cycle disorders. Injectable contraceptives every 3 months have a high complaint rate when compared to other contraceptives. This is because the hormonal imbalance in the body becomes larger and causes menstrual cycle disorders to occur more frequently and bleeding more when compared to other contraceptives.

The number of women participating in sports and physical activity continues to increase. Although exercise has many advantages, it can cause some disruption to female workers if done excessively. Physical activity of heavy intensity can cause physiological disorders of the menstrual cycle. presence of menstruation (amenorrhoea), thinning of the bones (osteoporosis), menstrual irregularities or intermenstrual bleeding, abnormal growth of the uterine wall, and infertility (Anderson, 1965; Mittleman et al, 1993; Moisan, 1991). The nature and severity of symptoms depend on several things such as the type of exercise, the intensity and duration of training, and the rate at which the trainer program is progressing

Women who participate in competitive sports have a higher risk of developing or developing eating disorders, menstrual cycle irregularities and osteoporosis, known as the Female worker Triad. Excessive exercise can lead to disorders of hypothalamic dysfunction leading to impaired GnRH secretion. This causes delayed menarche and menstrual cycle disorders. The main factor causing GnRH suppression of female female workers is the excessive use of energy that exceeds the energy intake of the female worker. Nutritional deficiency is a factor in the hypoestrogen state in female female workers. 
Harlow \& Matanoski (1991). Suggest that the association between weight, physical activity, and stress and variation in the length of the menstrual cycle. The risk factors for physical activity in training for prolonged menstrual cycles are associated with the initiation of high-volume aerobic exercise, and are specific to the principle of specificity training. Other sources describe continuous physical activity with aerobic energy sources and only requiring light intensity to potentially increase the risk of menstrual cycle disorders than vigorous anaerobic exercise followed by repetitions.

The mechanisms associated with the physiological processes of the body during exercise with reproduction are as follows: (1) Endocrinological adaptation, for example: hypothalamic dysfunction occurs, as well as inhibition of GnRH pulsation, (2)The body's adaptation to energy consumption decreases the hormone leptin during exercise, (3)Metabolic adaptation, causing changes in skeletal muscle mass and bone density.

\section{Conclusion}

Several studies have noted that menstrual disruption occurs during the period of employment where weight loss is significantly more at risk of delayed menstruation than female workers who maintain their weight during the testing period. Women who participate in competitive sports have a higher risk of developing or developing eating disorders, menstrual cycle irregularities and osteoporosis, known as the Female Female worker.

\section{References}

Anderson, T. W. (1965). Swimming and exercise during menstruation. Journal of Health, Physical Education, Recreation, 36(8), 66-68.

Arrais, R. F., \& Dib, S. A. (2006). The hypothalamus-pituitary-ovary axis and type 1 diabetes mellitus: a mini review. Human reproduction, 21(2), 327-337.

Bushman, B., Masterson, G., \& Nelsen, J. (2006). Anaerobic power performance and the menstrual cycle: eumenorrheic and oral contraceptive users. Journal of sports medicine and physical fitness, 46(1), 132.

Côté, I., Jacobs, P., \& Cumming, D. (2002). Work loss associated with increased menstrual loss in the United States. Obstetrics \& Gynecology, 100(4), 683-687.

Harlow, S. D., \& Matanoski, G. M. (1991). The association between weight, physical activity, and stress and variation in the length of the menstrual cycle. American journal of epidemiology, 133(1), 38-49.

Huang, T. S., Wang, Y. H., Lai, J. S., Chang, C. C., \& Lien, I. N. (1996). The hypothalamuspituitary-ovary and hypothalamus-pituitary-thyroid axes in spinal cord-injured women. Metabolism, 45(6), 718-722.

Iyibozkurt, C. (2011). Control of the menstrual cycle: the impact of nutrition on reproductive function. Turkish Archives of Pediatrics, 46(Supp: 1), 107-110.

Kissell, K. A., Danaher, M. R., Schisterman, E. F., Wactawski-Wende, J., Ahrens, K. A., Schliep, K., ... \& Mumford, S. L. (2014). Biological variability in serum anti-Müllerian hormone throughout the menstrual cycle in ovulatory and sporadic anovulatory cycles in eumenorrheic women. Human reproduction, 29(8), 1764-1772. 
Loucks, A. B., Laughlin, G. A., Mortola, J. F., Girton, L., Nelson, J. C., \& Yen, S. S. (1992). Hypothalamic-pituitary-thyroidal function in eumenorrheic and amenorrheic athletes. The Journal of Clinical Endocrinology \& Metabolism, 75(2), 514-518.

Mittleman, M. A., Maclure, M., Tofler, G. H., Sherwood, J. B., Goldberg, R. J., \& Muller, J. E. (1993). Triggering of acute myocardial infarction by heavy physical exertion-protection against triggering by regular exertion. New England Journal of Medicine, $329(23), 1677-1683$.

Moisan, J. O. C. E. L. Y. N. E., Meyer, F. R. A. N. C. O. I. S., \& Gingras, S. U. Z. A. N. N. E. (1991). Leisure physical activity and age at menarche. Medicine and science in sports and exercise, 23(10), 1170-1175.

Ng, T. P., Foo, S. C., \& Yoong, T. (1992). Menstrual function in workers exposed to toluene. Occupational and Environmental Medicine, 49(11), 799-803.

Nohara, M., Momoeda, M., Kubota, T., \& Nakabayashi, M. (2010). Menstrual cycle and menstrual pain problems and related risk factors among Japanese female workers. Industrial Health, 1012100044-1012100044

Norman, R. (2008). The human menstrual cycle. In The active female (pp. 123-129). Humana Press.

Su, S. B., Lu, C. W., Kao, Y. Y., \& Guo, H. R. (2008). Effects of 12-hour rotating shifts on menstrual cycles of photoelectronic workers in Taiwan. Chronobiology international, 25(2-3), 237-248.

Zhao, M. X., Zhou, G. Y., Zhu, J. Y., Biao, G. O. N. G., Hou, J. X., Tong, Z. H. O. U., ... \& Yue, B. A. (2015). Fluoride exposure, follicle stimulating hormone receptor gene polymorphism and hypothalamus-pituitary-ovarian axis hormones in Chinese women. Biomedical and Environmental Sciences, 28(9), 696-700. 